\title{
ARDUINO UNO BASED OBSTRUCTIVE SLEEP APNEA DETECTION USING RESPIRATORY SIGNAL
}

\author{
Agnel John K.J' ${ }^{1}$, Pamela .D ${ }^{2}$ \\ ${ }^{I}$ M.Tech Embedded Systems, Karunya University \\ ${ }^{2}$ Assistant professor, Karunya University
}

\begin{abstract}
The monitoring of breathing dynamics is an essential diagnostic tool in various clinical environments, such as sleep analysis, intensive care and central nervous and physiological disorder analysis. This paper introduces a mathematical representation of respiratory pattern in frequency domain. Sleep apnea is defined as cessation of airflow to the lungs during sleep for 10 sec. It normally results from either lack in neural input from the central nervous system (Central Sleep Apnea) or Upper airway collapse (Obstructive sleep apnea).Microcontroller based sleep apnea monitor consists of a piezoelectric sensor attached to rib cage of patient. The amplified signal obtained from the patient is applied to the microcontroller. The method mentioned in the paper is based on extraction of four enhanced main energy features of respiratory signal from 30 second respiratory data through auto regressive modeling and other techniques. The four features extracted are Signal power, Respiration frequency, Dominant frequency in power spectrum, Maximum power in specturm. These features are compared with their threshold values and introduced to a series of condition for each epoch.
\end{abstract}

Keywords: Auto-regression, Sleep apnea, Energy index, Respiratory frequency, Least squares method.

\section{INTRODUCTION}

Sleep is essential for humans although its basic physiological function remains obscure .Respiration monitoring allows the continuous measurement and analysis of breathing dynamics and thus the detection of various disorders. Sleep apnea is defined as cessation of airflow to the lungs during sleep for $10 \mathrm{sec}$ or more . In order to measure the respiration certain things have to be ignored such as noise in the measuring device and artifacts obtained by the body movements. One can think about a multitude classification algorithm that could help to reach better identification mechanism. Such as classifying different types of signals with different information with different characteristic features .There have been tremendous growth in the area of development of classification algorithm for respiration signal.

OSA is most common form of sleep apnea, Symptoms of OSA are Fatigue, Daytime sleepiness, and Heart related problems and system. The gold standard diagnostic test for obstructive sleep apnea (OSA) is overnight polysomnography (PSG) which is costly in terms of time and money. As a result, primary care providers may be reluctant about ordering polysomnography and patients unwilling to attend their tests. Many patients who suffer from sleep apnea do not get proper diagnosis due to the discomfort and complicacy of this current diagnosis system.

Sleep apnea syndrome can be detected by the analysis of respiration signals, In medical terms two categories are used to describe the grade of this sleep disorder. Hypopnea and apnea. Hypo apnea cannot be due to ceased respiration movements, while apnea the respiration movement is totally ceased less than $5 \%$ of the average physiological range.
The methods used to treat OSAS are weight loss, surgical widening of the airway, and mechanical devices for upper airway maintenance, electrical stimulation of the upper airway, pharmacological agents, and oxygen administration. All of these methods seem to have some benefits. Presently, the most popular therapeutic method for treatment of OSA and hypopnea is the nasally applied continuous positive airway pressure (CPAP) method. A CPAP machine applies a constant pressure, called the prescribed pressure, to the patient's airway through a nasal mask while the patient sleeps. The level of applied pressure is determined in a sleep laboratory by manually titrating the applied pressure to eliminate all respiratory events (apnea, hypopnea and snoring) during sleep. Advantages of nasal CPAP are that it produces immediate relief, is noninvasive, and can be used while achieving weight loss or considering surgical treatment.

Major disadvantage of CPAP process is that irrespective to the variations in patients weight, alcohol consumption, nasal congestion and sleeping position its forced to apply uniform pressure throughout the night .This will cause uneasy because of this increased expiratory effort or sensation of forced air throughout his nostrils .Fuzzy logic can be implemented which can be increased decision making process in computing. Sleep apnea is a difficult condition to describe with deterministic mathematical method. Fuzzy logic is well suited when certain areas of defining quantities overlap or vary person by person.

Our proposed work shows a method for classifying the respiratory signal using microcontroller. The capability of classifying respiratory signals and detecting apnea episodes are of crucial importance for clinical purposes. It describes 
an automatic classification algorithm using features derived from the autoregressive modeling and modified threshold crossing schemes that was used to classify respiratory signals into the following categories: (1) Normal respiration, (2) Respiration with artifacts and (3) Sleep apnea. This classification is capable of detecting fatigue of the human by identifying sleep apnea, early detection of sleep troubles and disorders in groups at risk. The main contribution of this paper is the determine sleep apnea event occurred during sleep using a low cost instrument. Instrument thoroughly check the sleep intervals for a duration of 1 hour and predict the chances of apnea events.

\section{METHODOLOGHY}

\subsection{Signal Acquisition}

The main advantage of using respiratory signal is such that it will detect the case of non breathing stage very easily when compared with other means of analysis such as polysmography, ,ECG,EMG. The surface of respiratory signals is acquired for duration of 30 seconds using Lab VIEW and Biokit. From the results in [1], the respiratory signals alone are sufficient and perform even better than ECG, EEG and EMG. In our paper, we consider only the respiratory signal for the detection of sleep apnea since it is more convenient and do not require more number of electrodes on the skin. The human respiratory signal as shown in is classified into three major classifications namely.

1. Normal breathing

2. Sleep apnea.

\section{Normal Respiration}

The normal respiration is characterized by the presence of a certain rhythm and the presence of some energy level in the signal.

\section{Sleep apnea}

Apnea is easily classified as the absence of energy (ventilation activity) as well as a lack of rhythm. The respiration rate was below a critical level.

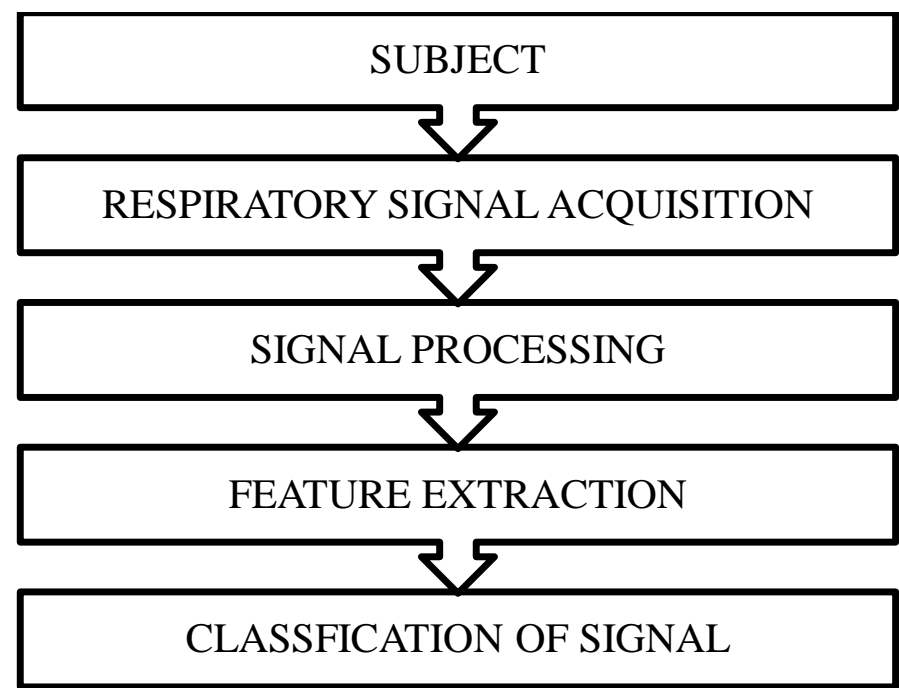

Motion artifact
Motion Artifact is generally characterized by a sudden increase in the amplitude of the signal and by a sudden variation in the rhythm of the heart usually has the higher energy when compared to the normal respiration. Motion artifacts are transient baseline changes caused by changes in the skin impedance. This type of interference represents an abrupt shift in base line due to movement of the patient while the respiratory signal is being recorded.

\subsection{Block Diagram}

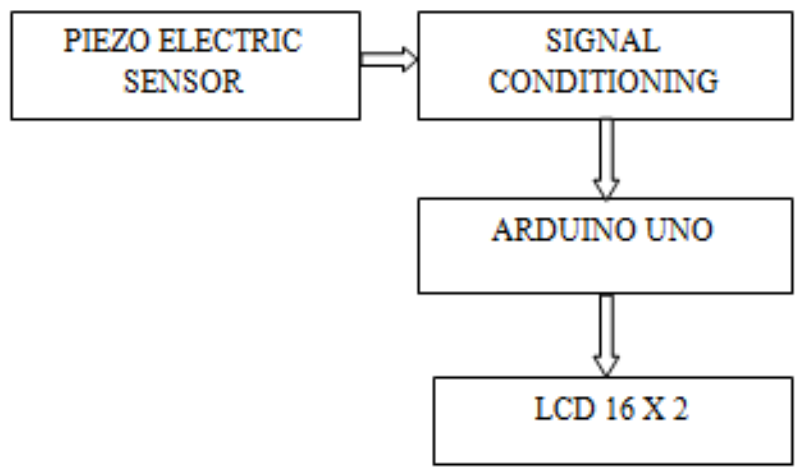

Fig 2.2.1 Block Diagram

\subsection{Feature Extraction}

Due to its complex nature the proper selection of features are essential for classification. Many researchers uses time domain, frequency domain, time-frequency domain techniques for classification of signals.In this work the feature extracted are Energy index(EI), Respiration Frequency(FZX),Dominant Frequency(FAR), Strength of dominant Frequency(STR).These features are extracted for every movements and the calculation is given below.

\subsubsection{Power of the Signal (Ps):}

For a continuous time signal $\mathrm{F}(\mathrm{t})$, the energy contained over a finite interval(T2>T1) is defined as follows .Energy index is defined as the energy present per sample acquired by the DAQ. For non breathing condition the sample energy will be very low when compared energy obtained during breathing state .1

$$
\mathrm{Ps}=\frac{1}{\mathrm{~N}} \quad \sum_{0}^{\mathrm{N}-1}|\mathrm{X}(\mathrm{n})|^{2}
$$

where $N$ is the length of the signal and $X(n)$ represents the respiratory signal in samples

\subsubsection{Respirational Frequency (RF).}

Since we are acquiring a random signal it's not possible to calculate the respiration frequency directly. This is due to the reason that DC baseline keep on changing with time .This can be solved by using Zero crossing algorithm for the estimation of the frequency in white noise[a]. Zero-crossing is a commonly used term in electronics, mathematics, and image processing. In mathematical terms, a "zero-crossing" is a point where the sign of a function changes represented by a crossing of the axis. Respiration frequency (RF) can be 
determined by counting the number of times that $x(n)$ crosses a baseline which is defined as the square root of EI.

$$
\mathrm{RF}=\sqrt{\mathrm{Ps}}
$$

\subsubsection{Dominant Freq in Power Spectrum (DF)}

Respiratory signal comes under different types of random signal. In order to obtain the power spectrum of signal in frequency domain we make use auto regression analysis. Signal is then represented as AR model. Here we make use of least square estimation method to obtain AR coefficients. After plotting the power spectrum we obtain frequencies along $\mathrm{X}$ axis and corresponding power along $\mathrm{Y}$ axis. Dominant frequency is defined as the frequency at which we obtain maximum power.a1 and a 2 are the AR coefficients

$$
\mathrm{DF}=\frac{\mathrm{Fs}}{2 \pi} \arctan \frac{\mathrm{a} 01}{\mathrm{a} 02}
$$

\subsubsection{Maximum Power in Power Spectrum (MP)}

Strength of dominant frequency is defined as the maximum power obtained per the given samples, As we know that at dominant frequency we obtain maximum power, so the $\mathrm{Y}$ axis reading for that particular frequency is considered as STR. This can be obtained by AR coefficient by using the formula

$$
\mathrm{MP}=\sqrt{a 1^{2}+a 2^{2}}
$$

Basically, DF and MP serve the same purpose as power spectrum usually does, indicating the dominant frequency and its corresponding power.. The degree of reliability of the respiration rate estimate was determined by MP, which have a value between 0 and 1 . For very regular rhythm, MP is very close to 1 (as in the case of normal respiration). If MP is too low, then the rate estimates DF and RF are deemed to be less.

\subsection{LabVIEW Simulation Results}

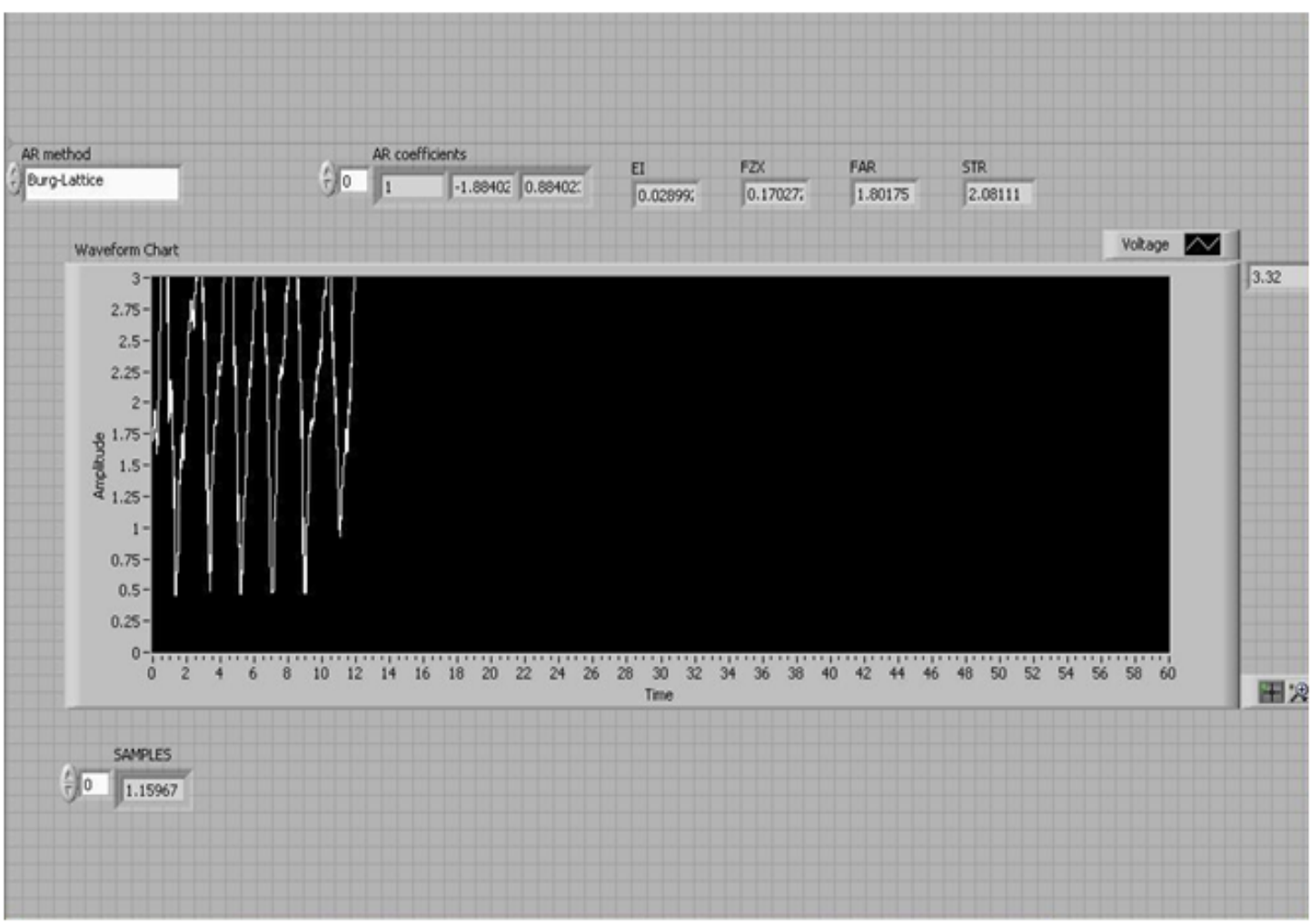

Fig 2.3.1 Acquired signal for a duration of 30 seconds breathing 


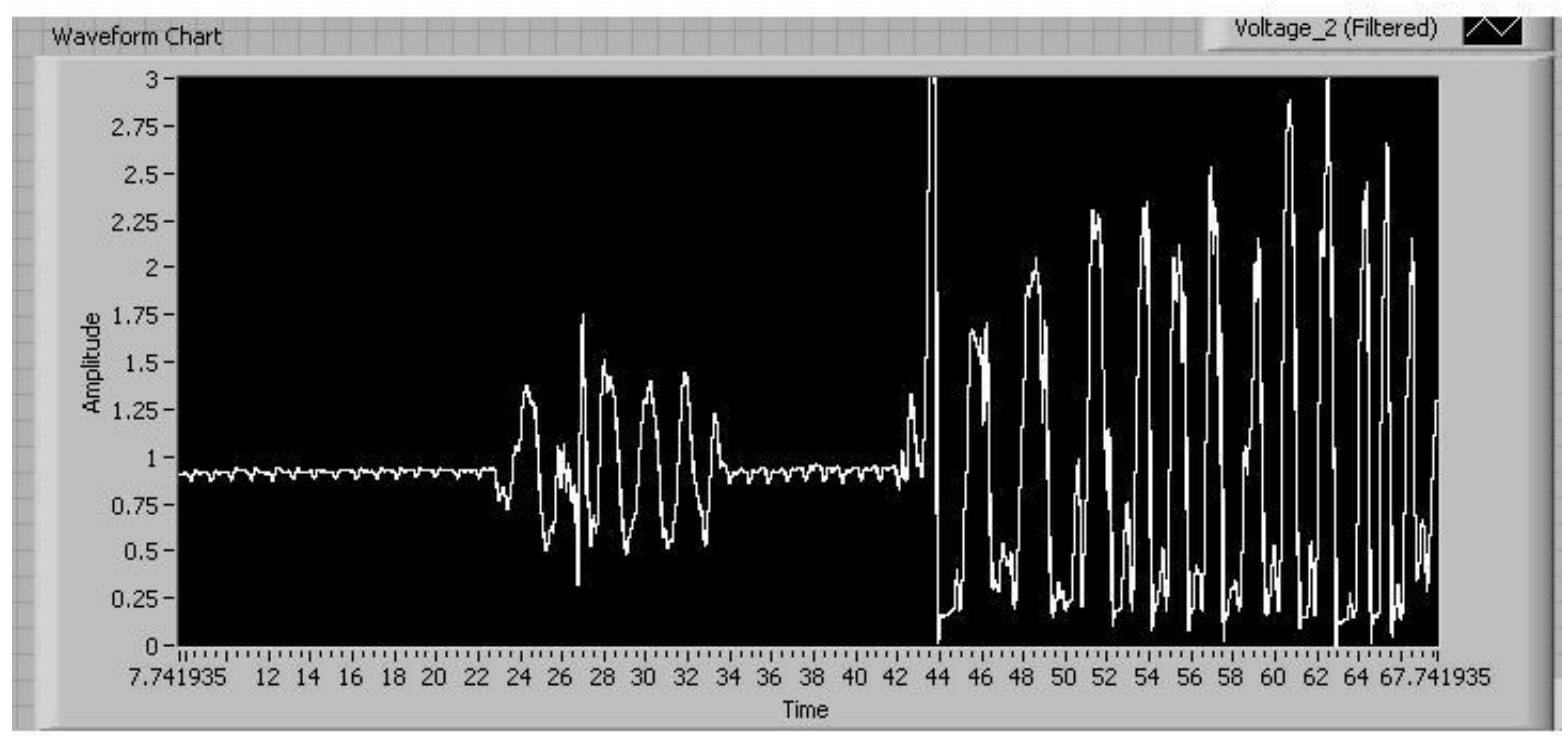

Fig 2.3.2 Acquired signal for a duration of 30 seconds non breathing

Acquiring respiratory signals for a sleep apnea patient seems to be difficult. Hence normal persons respiratory signals are acquired by forcing him to stop breathing for duration of 10 sec and continuing breathing which can be considered as equivalent to respiratory signals of a sleep apnea patient .

\section{Simulation Results}

Table 2.3.1 Simulation results for sample 1.

\begin{tabular}{|l|l|l|}
\hline No & Episodes & Simulation \\
\hline 1 & Apnea & 7 \\
\hline 2 & Normal & 52 \\
\hline
\end{tabular}

Table 2.3.2 Simulation results for sample 2 .

\begin{tabular}{|l|l|l|}
\hline No & Episodes & Simulation \\
\hline 1 & Apnea & 652 \\
\hline 2 & Normal & 428 \\
\hline
\end{tabular}

\subsection{Hardware Results:}

Arduino uno board is used to detect respiratory signal samples from the patient. Piezo electric sensor attached to the wing part of the patient detects change in voltage with in a range of $0-5 \mathrm{~V}$ When ever patients exhales chest part is expanded and this change in pressure is detected by the piezoelectric sensor and converted into digital values by using 10 bit ADC in Arduino development board.

Table 2.4.1 Threshold obtained by hardware

\begin{tabular}{|l|l|l|l|l|l|l|}
\hline $\begin{array}{l}\text { Sampl } \\
\text { e }\end{array}$ & $\begin{array}{l}\text { Ps_ } \\
\text { L }\end{array}$ & $\begin{array}{l}\text { Ps_ } \\
\mathbf{H}\end{array}$ & $\begin{array}{l}\text { DF_M } \\
\mathbf{i}\end{array}$ & $\begin{array}{l}\text { DF_M } \\
\mathbf{x}\end{array}$ & $\begin{array}{l}\text { MP } \\
\mathbf{L}\end{array}$ & $\begin{array}{l}\text { MP } \\
\mathbf{H}\end{array}$ \\
\hline $\begin{array}{l}\text { Norma } \\
\text { l }\end{array}$ & 1.08 & 2.5 & .36 & .65 & 1.70 & 3.41 \\
\hline Apnea & .04 & .20 & 1.43 & 3.3 & .30 & .61 \\
\hline
\end{tabular}

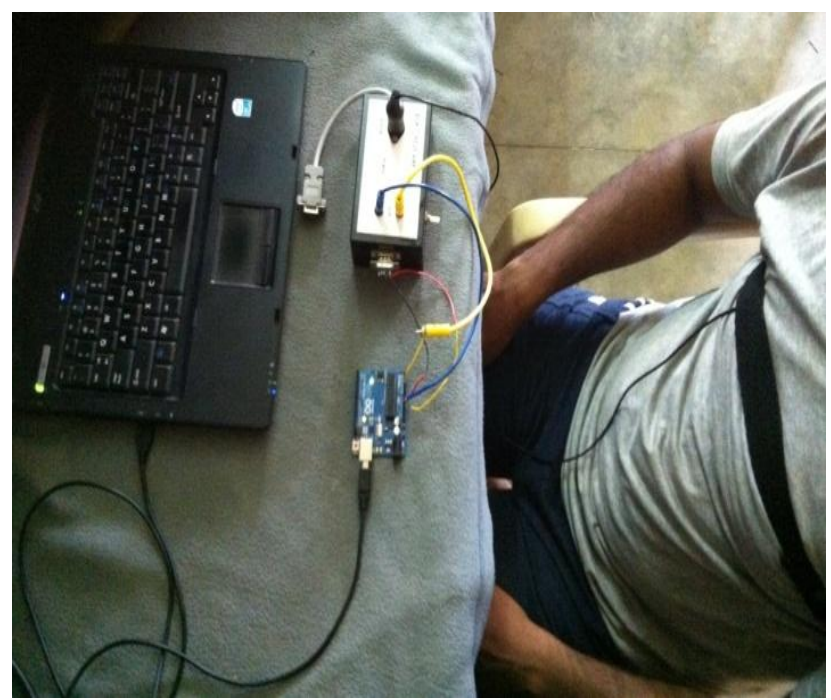

After obtaining the energy features , min \& max thresholds for each of the feature is being calculated from the standard given. This obtained thresholds are then used in the automatic classification algorithm for distinguishing sleep apnea events from normal breathing events. Process is being carried out through out the sleep and when ever there is a state of non breathing with in the duration of $15 \mathrm{sec}$, apnea count get updated. And if there is condition like the apnea event have been recorded for continuous two $15 \mathrm{sec}$ duration processing then alert will be activated indicating that the patient had stopped breathing not safely.

Table 2.4.2 Event count for a duration of $5 \mathrm{~min}$

\begin{tabular}{|l|l|l|}
\hline No & Episodes & Simulation \\
\hline 1 & Apnea events & 3 \\
\hline 2 & Normal events & 27 \\
\hline
\end{tabular}




\section{CONCLUSION \& FUTURE EXPANSION}

This work can be developed and implemented in real time application for detecting sleep apnea. To develop this project in real time, we have to design a processor and the algorithm should be improved by adding calibration procedures and is adjusted to run on ARM CORTEX. The electrical signals which are analog in nature should be converted into digital by analog to digital converter (ADC) and is given to the ARM 9. Then the processor will process and detect the appropriate signal. Graphical LCD 84X64 or $\mathrm{PC}$ monitor can be used to display the name of the signal.

\section{REFERENCES}

[1] A.Bhavani Sankar,D.Kumar, K.Seethalakshmi, " Enhanced Method for Extracting Features of Respiratory Signals and Detection of Obstructive Sleep Apnea Using Threshold Based Automatic Classification Algorithm ". International Journal of Computer Science \& Emerging Technologies (EISSN: 2044-6004) 38Volume 1, Issue 4, December 2010

[2] Walter Karle, Claudio Mattiussi and Dario Florea," Sleep and Wake Classication With ECG and Respiratory Effort Signals" IEEE Trans-actions on Biomedical Circuits and Systems,2009

[3] Paul bourke,"Auto regression analysis", ieee transactions on instrumentation and measurement, vol. 52, no. 1, february 2003

[4] Vladmir Friedman ," A Zero crossing algorithm for the estimation of the frequency of a single sinusoid with white noise'IEEE transaction on signal processing, VOL - 42, No6, JUNE 1994

[5] Taikang Ning and Joseph D. Bronzino , “Automatic classification Of respiratory signals"IEEE engineering in Medicine and Biology Soceity 11 th annual international conference $-0669 \mathrm{CH} 2770$ 6/89/0000-0669 ---- 1989

[6] Peter vardy,szcholas bangar, "Detection of airway obstruction and sleep apnea by analysing the phase relation of respiration movement signals"IEEE transaction on instrumentation and measurement, VOL -52, No 1, Feb - 2003

[7] Lorena S. Correa, Eric Laciar, Member IEEE, Vicente Mut, Abel Torres, Member IEEE, and Raimon Jané, Member IEEE," Sleep Apnea Detection based on Spectral Analysis of Three ECG Derived Respiratory Signals,"31st Annual International Conference of the IEEE EMBS Minneapolis, Minnesota, USA, September 2-6, 2009

[8] Stephen J Redmond and Conor Henegan,"Cardio respiratory based sleep staging in subjects With obstructive sleep apnea",IEEE Transaction on Biomedical Engineering, VOL-53, NO - 3, 2006. 